CLINICAL STUDY

\title{
The utility of basal serum LH in prediction of central precocious puberty in girls
}

\author{
Yehonatan Pasternak $^{1}$, Michael Friger ${ }^{2}$, Neta Loewenthal ${ }^{1}$, Alon Haim ${ }^{1}$ and Eli Hershkovitz ${ }^{1}$ \\ ${ }^{1}$ Pediatric Endocrinology and Metabolism Unit, Faculty of Health Sciences, Soroka Medical Center, Ben-Gurion University of the Negev, PO Box 151, \\ IL-84101 Beer-Sheva, Israel and ${ }^{2}$ Department of Epidemiology and Health Services Evaluation, Faculty of Health Sciences, Ben-Gurion University of the \\ Negev, Beer-Sheva, Israel \\ (Correspondence should be addressed to E Hershkovitz; Email: elih@bgu.ac.il)
}

\begin{abstract}
Objective: The mainstay of distinction between prepubertal girls and girls who are suspected of having central precocious puberty (CPP) is based on gonadotropin measurements after a $\mathrm{GnRH}$ stimulation test to evaluate hypothalamic-pituitary-gonadal axis maturity. The objective of this study was to determine whether a single basal gonadotropin measurement carries a useful predictive value in verifying or refuting the diagnosis of CPP.

Design and methods: Basal serum LH and FSH were measured by a chemiluminescent immunometric assay in a cohort of girls who had been evaluated for CPP before and after GnRH stimulation test. Peak LH levels higher than $5 \mathrm{IU} / \mathrm{l}$ were considered a pubertal response.

Results: Eighty girls with suspected breast development before 8 years of age were enrolled to the study, out of whom 42 had CPP.

Low basal serum LH $(\leq 0.1 \mathrm{IU} / \mathrm{l})$ was sufficient to rule out the diagnosis of CPP in $94.7 \%$ of the 38 prepubertal girls; the sensitivity of basal LH levels for this purpose was only $64 \%$. The basal FSH and the basal LH to FSH ratio achieved less efficient predictive value with 76 and $71 \%$ sensitivity and 73 and $86 \%$ specificity respectively.

Conclusion: A single basal LH measurement may be adequate to confirm but not to refute the presence of CPP in most of the girls who are evaluated for early pubertal signs.
\end{abstract}

European Journal of Endocrinology 166 295-299

\section{Introduction}

Precocious puberty is a phenomenon defined as development of pubertal signs before 8 years in girls and 9 years in boys. The signs include breast development, pubic hair, and menarche in girls, and genital enlargement and pubic hair in boys. The characteristics of puberty are normal but occur at a chronologically earlier age than expected.

The causes of precocious puberty divide into either central or peripheral mechanisms. Central precocious puberty (CPP) is a precocious puberty caused by the hypothalamic-pituitary-gonadal (HPG) axis activation. The phenomenon is much more common in girls than in boys $(1,2)$.

During normal puberty, inhibitory mechanisms affecting pulsatile GnRH secretion decline, which leads to increased pulsatile LH and FSH secretion. Pubertal LH secretion is characterized by high levels of peak LH secretion, first at night and later during the day. This secretion leads to higher levels of sex hormones in pubertal than in prepubertal subjects, and eventually to the appearance of pubertal signs and accelerated growth (3).
The diagnosis of CPP is done by documentation of an activated HPG axis in a child younger than normal pubertal age. Since there is a technical difficulty measuring GnRH levels directly, the diagnosis is focused at identifying pubertal serum levels of gonadotropins (LH and FSH) and sex hormones $(1,3)$. The standard method to verify HPG activity is the gonadotropin response to $\mathrm{GnRH}(4)$, or $\mathrm{GnRH}$ analog (GnRHa) administration (5).

With the development of newer and more sensitive immunoassays measuring serum gonadotropins, it was hypothesized that measuring basal gonadotropins will allow discrimination between pubertal and prepubertal values in the diagnosis of CPP. Lee et al. (6) used third generation assays (Architect and Delfia) for measuring basal serum LH levels in a small group of girls $(n=55$, 21 of whom were diagnosed as CPP) who were evaluated for clinical suspicion of CPP, and found that in most of the cases this single measurement was sufficient for the diagnosis of CPP.

Using a larger group of girls and a single different chemiluminescent assay, Immulite 2000, we hypothesized that a single basal gonadotropin measurement will be sufficient to distinguish the pubertal from 
prepubertal HPG axis in most cases. In this report, basal serum levels of LH and FSH were evaluated in patients for whom the diagnosis of CPP was verified or refuted using the current method of $\mathrm{LH}$ response to GnRH stimulation consistent with clinical signs of progressive pubertal development, accelerated growth rate, and skeletal bone age advancement. Using this method as the gold standard, the prognostic value of single basal gonadotropin levels for diagnosis of CPP was examined.

\section{Subjects and methods}

Subjects for this report were a cohort of girls who visited the pediatric endocrinology outpatient clinic due to suspected onset of pubertal signs such as breast budding before 8 years of age, and were evaluated using a GnRH stimulation test. All subjects underwent GnRH test before 9 years of age, and reported onset of pubertal signs during the 12 months before the test. Evaluation was made by a pediatric endocrinologist, and included: documentation of full medical history; onset and progression of pubertal changes, including Tanner breast and pubic hair stage; height and weight; growth velocity; radiographic skeletal age documentation; and GnRH stimulation test.

Girls with suspected breast development were included in the study. The girls were divided into two groups according to the GnRH stimulation test results. Girls who had a peak LH values $\geq 5 \mathrm{IU} / \mathrm{l}$ were considered as having a pubertal activation of the HPG axis and therefore as having $\operatorname{CPP}(4,6)$. The girls who had lower values were considered prepubertal.

Eighty girls were enrolled for the study, 42 of whom had CPP (mean age: $7.5 \pm 1.34$ and $7.04 \pm 1.16$ years for CPP and prepubertal groups respectively; $P=0.11$ ). Height SDS and skeletal age advancement (bone age (BA) - chronological age (CA)) were similar between the groups: height SDS $0.83 \pm 0.95$ vs $0.72 \pm 0.93$ and BA-CA $1.38 \pm 1.4$ vs $1.06 \pm 1.49$ for the CPP and prepubertal groups respectively. Ovarian volume $\left(\mathrm{ml}^{3}\right)$, as determined by abdominal ultrasound was larger in CPP girls $(n=12)$ than in prepubertal girls $(n=15)$ : $3.5 \pm 2.09$ vs $2.12 \pm 2.1$ respectively $(P=0.03)$.

The clinical course of the CPP group (42 girls) after the GnRH stimulation test was variable. Ten girls were treated with GnRHa (decapeptyl), changing the course of their natural development, and were followed up for 1-4.2 years. Out of the untreated girls, 23 have continued to show progressive pubertal development during the 4-25 months of follow-up after the $\mathrm{GnRH}$ test. However, the pubertal status of nine girls regressed and progression of pubertal signs was not found in the next 0.5-6.5 years follow-up after the test.

In the prepubertal group (38 girls), 30 girls did not show pubertal signs up to 0.3-4.2 years of follow-up at the ages of 6-11.5 years, while eight girls have shown signs of breast development, six of them after 9 years of age, and two before 9 years of age ( 8.4 and 8.8 years). The follow-up after the test was conducted for 3-51 months.

The study was approved by the local ethics institutional review board of Soroka Medical University Center, Beer-Sheva.

\section{GnRH test}

Blood samples were drawn before i.v. administration of $0.1 \mathrm{mg}$ Gonadorelin (GnRH, Hoechst, Frankfurt, Germany) and at $15,30,45$, and 60 min after. All the tests were conducted between 0800 and $1000 \mathrm{~h}$.

LH was measured by a chemiluminescent immunometric assay (Immulite 2000, Diagnostic Products Corporation, Los Angeles, CA, USA). The calibration range of the assay was up to $200 \mathrm{IU} / \mathrm{l}$, with an analytical sensitivity of $0.05 \mathrm{IU} / \mathrm{l}$ and functional sensitivity accounted as the $20 \%$ coefficient of variation (CV) level was $0.1 \mathrm{IU} / \mathrm{l}$. The intra-assay CV values were $13.1,3.04,3.71$, and $3.6 \%$ at the levels of $0.15,1.04$, 1.89 , and $8.7 \mathrm{IU} / \mathrm{l}$ respectively. The corresponding inter-assay $\mathrm{CV}$ values were 23.9, 6.6, 6.2, and 6.7\%. The cross-reactivity with human chorionic gonadotropin was $0.20 \%$. FSH was measured by an immunometric assay (Immulite 2000, Diagnostic Products Corporation). The calibration range of the assay was up to $170 \mathrm{IU} / \mathrm{l}$, with an analytical sensitivity of $0.1 \mathrm{IU} / \mathrm{l}$. The intra-assay $\mathrm{CV}$ values were $2.5,2.9$, and $2.1 \%$ at the levels of $4,9.1$, and $40 \mathrm{IU} / \mathrm{l}$. The corresponding inter-assay $\mathrm{CV}$ values were $6.3,5.5$, and $4.3 \%$. The cross-reactivity with TSH was $0.01 \%$.

\section{Data analysis}

Means, maximal, and minimal values fit by pubertal status (with CPP or without CPP) for each gonadotropin were found, and the means were compared by independent sample $t$-test. Binary logistic regression models were fit with pubertal status using stimulated peak LH results as the outcome and the non-stimulated gonadotropin value ( $\mathrm{LH}, \mathrm{FSH}$, and $\mathrm{LH} / \mathrm{FSH}$ ratio) as the

Table 1 Descriptive statistics of the basal non-stimulated sample of $\mathrm{LH}, \mathrm{FSH}$, and $\mathrm{LH} / \mathrm{FSH}$ ratios in girls with CPP and prepubertal girls.

\begin{tabular}{llcr}
\hline $\begin{array}{l}\text { Group/ } \\
\text { hormone }\end{array}$ & $\boldsymbol{n}$ & Mean \pm s.d. & $\begin{array}{c}\text { Range } \\
\text { (min-max) }\end{array}$ \\
\hline CPP & & & \\
LH (IU/I) & 42 & $0.87 \pm 1.28$ & UD-5.9 \\
FSH (IU/I) & 42 & $3.5 \pm 1.6$ & $1.3-6.5$ \\
LH/FSH ratio & 42 & $0.19 \pm 0.25$ & $0-0.97$ \\
Prepubertal & & & \\
LH (IU/I) & 38 & $0.04 \pm 0.05$ & UD-0.2 \\
FSH (IU/I) & 38 & $1.69 \pm 1.08$ & UD-4.0 \\
LH/FSH ratio & 38 & $0.019 \pm 0.03$ & $0-0.1$ \\
\hline
\end{tabular}

UD, undetected. 


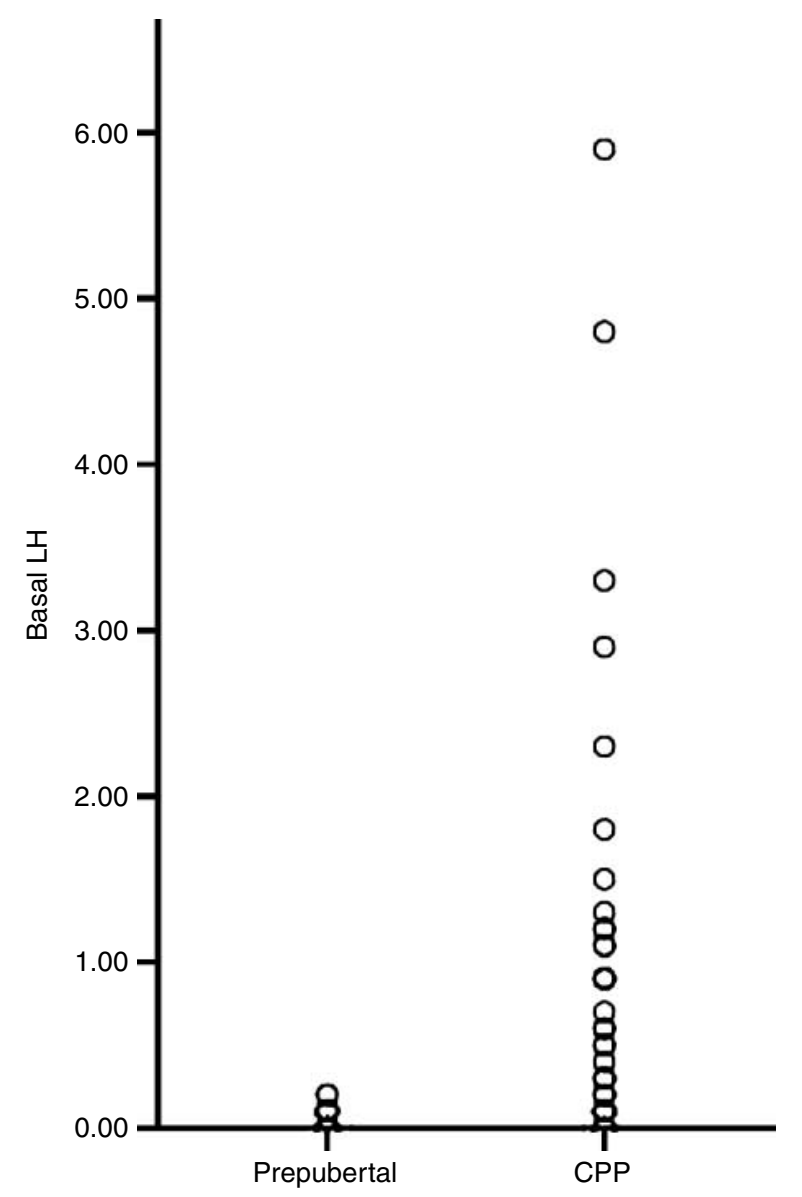

Figure 1 Basal serum LH levels. Thirty-six of the 38 prepubertal girls had basal serum LH levels $\leq 0.1 \mathrm{IU} / \mathrm{l}$.

regressor. Using the logistic regression models, receiver operating curves (ROCs) were constructed to evaluate the sensitivity and specificity at each level of gonadotropin based on predicted probability, and area under the curve (AUC) was measured for each curve. Youden's J index ((sensitivity + specificity $)-1)$ was used to determine the optimal gonadotropin cut off point from the ROC for each assay to differentiate girls with CPP from prepubertal girls (7). For this cut off point, specificity and sensitivity were then re-calculated to evaluate the cut off point efficacy. $P$ value $<0.05$ was considered statistically significant. Statistical analyses were performed by SPSS 17 Statistical Software (IBM Corporation, Chicago IL, USA).

\section{Results}

The descriptive statistics for basal gonadotropins (LH, FSH, and LH/FSH ratio) divided into CPP and non-CPP groups are displayed in Table 1. The basal LH, FSH, and LH/FSH ratio means were significantly higher in the CPP group than in the prepubertal group $(P<0.01)$.
However, considerable overlapping was noted between the two groups: 18,29 , and nine girls with CPP had a basal serum LH, FSH, and LH/FSH ratio, respectively, within the basal serum range of the prepubertal girls. It is important to note that 36 of the 38 prepubertal girls $(94.7 \%)$ have demonstrated undetectable or $\leq 0.1 \mathrm{IU} / \mathrm{l}$ values for basal LH (Fig. 1). Fifteen of the $42 \mathrm{CPP}$ girls have also demonstrated basal LH values $\leq 0.1$ (35.7\%).

Three different models were performed to determine a cut off point for the diagnosis of CPP using a nonstimulated gonadotropin sample, based on the basal LH, $\mathrm{FSH}$, and LH/FSH ratio. ROCs were constructed for each model, and AUC was measured for each curve (Fig. 2). Maximum predictability was reached using the basal LH model with an AUC of 0.849 . The optimal gonadotropin cut off point for discriminating CPP from prepubertal girls was then determined for each gonadotropin based on sensitivity and specificity (Table 2). Two other models combining different gonadotropins (a: LH and FSH and b: LH, FSH, and LH/FSH ratio) did not provide a significant contribution and therefore are not shown in our analysis.

Maximal Youden's J index was reached based on specificity of $94.7 \%$ and sensitivity of $64.4 \%$ for the basal LH model; an LH cut off point of $0.1 \mathrm{IU} / \mathrm{l}$ was chosen to discriminate between CPP and prepubertal girls (i.e. girls with $\mathrm{LH}$ values $>0.1$ were considered to have $\mathrm{CPP}$ ). The positive predictive value (PPV) of such discrimination in a population of girls suspected of having CPP was 93.1\% (95\% confidence interval (CI) $0.87-0.98)$, while the negative predictive value was $70.5 \%$ (95\% CI 0.80-0.60). The FSH model yielded less favorable performances such as sensitivity of $76.2 \%$ and specificity of $73.3 \%$ for a cut off point of $2.25 \mathrm{IU} / \mathrm{l}$. The sensitivity and specificity for the LH/FSH ratio values were 71.4 and $86.8 \%$ respectively, for a cut off point of 0.05 .

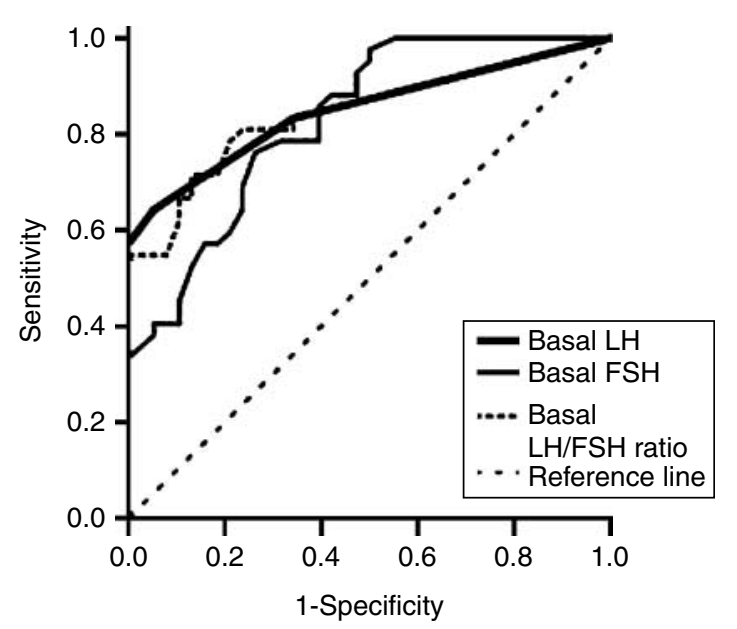

Figure 2 ROCs used to evaluate the sensitivity and specificity at each level for $\mathrm{LH}, \mathrm{FSH}$, and $\mathrm{LH} / \mathrm{FSH}$ ratio. 
Table 2 Sensitivity and specificity for selected gonadotropin cut off point values discriminating CPP from prepubertal girls.

\begin{tabular}{lccccc}
\hline Hormone & AUC $(95 \% \mathrm{Cl})$ & $\boldsymbol{P}$ & $\begin{array}{c}\text { CPP above this } \\
\text { cut off point }\end{array}$ & $\begin{array}{c}\text { Sensitivity } \\
(95 \% \mathrm{Cl})\end{array}$ & $\begin{array}{c}\text { Specificity } \\
(95 \% \mathrm{Cl})\end{array}$ \\
\hline LH $(\mathrm{IU} / \mathrm{I})$ & $0.849(0.762-0.935)$ & $<0.01$ & 0.1 & $0.64(0.59-0.70)$ & $0.94(0.89-0.98)$ \\
FSH (IU/I) & $0.829(0.742-0.916)$ & $<0.01$ & 2.25 & $0.76(0.66-0.86)$ & $0.73(0.64-0.83)$ \\
LH/FSH ratio & $0.843(0.755-0.930)$ & $<0.01$ & 0.05 & $0.71(0.62-0.81)$ & $86.8(0.79-0.94)$ \\
\hline
\end{tabular}

In addition, we re-evaluated the accuracy of peak LH $>5$ for diagnosis of CPP with a retrospective evaluation of the subjects' clinical data by a pediatric endocrinologist who determined the presence of CPP at least 2 years after the test was conducted, as the girls entered the normal pubertal age. A ROC was constructed in a similar manner to that mentioned above, and a cut off point of $4.9 \mathrm{IU} / \mathrm{l}$ was found to give the highest Youden's $\mathrm{J}$ index with a sensitivity and specificity of 78 and $79 \%$ respectively, reapproving the customary $5 \mathrm{IU} / \mathrm{l}$ cut off point.

\section{Discussion}

Based on the data presented here, a judicious use of a single basal serum (unstimulated) LH sample may be useful in girls with suspected precocious puberty due to apparent breast development, whose basal LH levels are higher than $0.1 \mathrm{IU} / \mathrm{l}$ in most cases (PPV 93.1\%), in the diagnosis of CPP. However, basal LH levels do not carry enough sensitivity to rule out CPP in girls whose basal LH levels are $\leq 0.1 \mathrm{IU} / \mathrm{l}$. When clinically indicated as evidenced by progression of pubertal signs, accelerated growth and BA advancement, a GnRH stimulation test should be applied for those girls to establish the diagnosis of CPP (Fig. 3). Basal levels of FSH were not proved to be useful in diagnosing CPP, and basal LH/FSH ratio does not seem to contribute significantly to the single basal LH results. Thus, a basal LH measurement can be obtained by the primary care physician, and if LH level $>0.1 \mathrm{IU} / \mathrm{l}$ is obtained, a diagnosis of CPP can be made, saving GnRH test inconvenience, costs, and time. However, applying this approach may lead to the diagnosis of CPP in a small number of true prepubertal girls. Therefore, careful judgment based on clinical follow-up and when required, repeated laboratory evaluation, including GnRH stimulation test, is essential for long-term management.

CPP as a clinical entity poses inherent diagnostic difficulties, since its signs are normal apart from the fact it appears earlier than normal. Moreover, the diagnosis is based on laboratory values and clinical evaluation, which can be misleading due to the increasing prevalence of juvenile obesity which is mistaken to be breast development. Basal LH levels might be especially helpful since the rise in gonadotropin levels is the first measurable step in the pubertal sequence of events, preceding the appearance of pubertal physical signs, growth spurt, and BA advancement. In addition, the tests in this study were conducted between 0800 and $1000 \mathrm{~h}$, a fact that minimizes the influence of diurnal fluctuations in gonadotropin values.

Previous studies on biochemical evaluation of girls with precocious puberty and its variants are relatively small (8) or based on multicenter studies using different hormone assays (9). Our results, carried out in a larger cohort, confirmed Lee et al.'s (6) findings of high specificity of a single basal LH sample and its usefulness for establishing the diagnosis of CPP. However, the sensitivity of a single basal serum LH sample was much lower in our study using an Immulite 2000 assay, in comparison to Lee et al.'s study, which used Architect and Delfia assays. This contradiction highlights the need for careful evaluation of each assay used for the diagnosis of CPP. The Immulite $2000 \mathrm{LH}$ assay showed good correlation to the Magia LH assay (10).

During the preparation of this manuscript, a study evaluating 88 girls with CPP compared with normal Danish girls' population reference values has been published. In that study, levels of basal LH were above two S.D. in about $70 \%$ of the girls over 7.5 years with CPP (11). This finding is similar to our sensitivity level of $64 \%$ of basal LH levels. This recent study demonstrated that basal LH levels were superior to FSH,

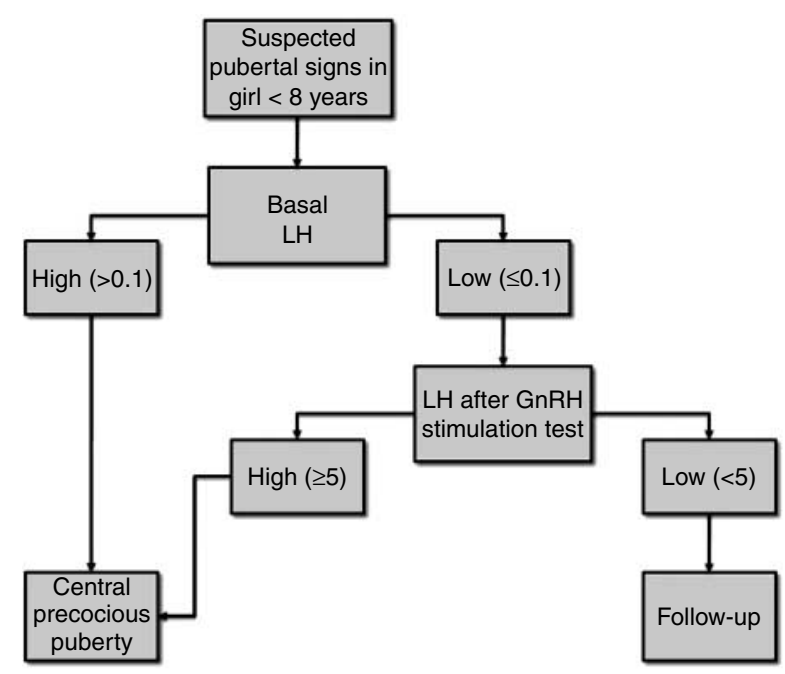

Figure 3 Proposed approach for diagnosis of CPP (LH levels are expressed in IU/I). 
estradiol, and inhibin B for the diagnosis of CPP. Mogensen et al.'s (11) study emphasizes the need to obtain normal baseline population data of gonadotropin levels. However, such data do not exist in most of the clinical centers that care for girls with CPP.

We conclude that measuring basal LH serum values has a role in the initial evaluation of girls with clinically suspected CPP, after evaluation of the performance of a specific LH local assay in a sample of girls suspected to have CPP.

\section{Declaration of interest}

The authors declare that there is no conflict of interest that could be perceived as prejudicing the impartiality of the research reported.

\section{Funding}

This research did not receive any specific grant from any funding agency in the public, commercial or not-for-profit sector.

\section{References}

1 Lee PA. Central precocious puberty, an overview of diagnosis, treatment and outcome. Endocrinology and Metabolism Clinics of North America 199928 901-911. (doi:10.1016/S0889-8529 (05)70108-0)

2 Rosenfield RL. Abnormal puberty Pediatric Endocrinology, pp. 483-490, Philadelphia: Saunders, 2002.

3 Iughetti L, Predieri B, Gallo C, Milioli S, Forese S \& Bernasconi S. Diagnosis of central precocious puberty: endocrine assessment Journal of Pediatric Endocrinology E Metabolism 200013 709-715. (doi:10.1515/JPEM.2000.13.S1.709)
4 Neely EK, Wilson DM, Lee PA, Stene M \& Hintz RL. Spontaneous serum gonadotropin concentrations in the evaluation of precocious puberty. Journal of Pediatrics 1995127 47-52. (doi:10. 1016/S0022-3476(95)70255-5)

5 Bhatia S, Neeli EK \& Wilson DM. Serum luteinizing hormone rises within minutes after depot leuprolide injection: implications for monitoring therapy. Pediatrics 2002109 e30. (doi:10.1542/ peds.109.2.e30)

6 Houk CP, Kunselman AR \& Lee PA. Adequacy of a single unstimulated leuteinizing hormone level to diagnose central precocious puberty in girls. Pediatrics 2008123 e1059-e1063. (doi:10.1542/peds.2008-1180)

7 Youden WJ. Index for rating diagnostic tests. Cancer 19503 32-35. (doi:10.1002/1097-0142(1950)3:1<32::AID-CNCR28 20030106 > 3.0.CO;2-3)

8 Choi JH, Shin YL \& Yoo HW. Predictive factors for organic central precocious puberty and utility of simplified gonadotropin-releasing hormone tests. Pediatrics International 200749 806-810. (doi:10. 1111/j.1442-200X.2007.02475.x)

9 Cisternino M, Arrigo T, Pasquino AM, Tinelli C, Antoniazzi F, Beduschi L, Bindi G, Borrelli P, De Sanctis V, Farello G, Galluzzi F, Gargantini L, Lo Presti D, Sposito M \& Tatò L. Etiology and age incidence of precocious puberty in girls: a multicentric study. Journal of Pediatric Endocrinology \& Metabolism 200013 (Suppl 1) 695-701. (doi:10.1515/JPEM.2000.13.S1.695)

10 Tello FL \& Hernández DM. Performance evaluation of nine hormone assays on the Immulite 2000® immunoassay system. Clinical Chemistry and Laboratory Medicine 200038 1039-1042. (doi:10.1515/CCLM.2000.154)

11 Mogensen SS, Aksglaede L, Mouritsen A, Sørensen K, Main KM, Gideon P \& Juul A. A diagnostic work-up of 449 consecutive girls who were referred to be evaluated for precocious puberty. Journal of Clinical Endocrinology and Metabolism 201196 1393-1401. (doi:10. 1210/jc.2010-2745)

Received 15 August 2011

Revised version received 10 October 2011

Accepted 14 November 2011 